\title{
Changes in Migration Matrices and Credit VaR - a new Class of Difference Indices
}

\author{
Stefan Trück ${ }^{\mathrm{a}, *}$, Svetlozar T. Rachev ${ }^{\mathrm{b}, \star}$ \\ ${ }^{a}$ Universität Karlsruhe, Germany and \\ Queensland University of Technology, Australia \\ ${ }^{\mathrm{b}}$ Universität Karlsruhe, Germany and \\ University of California, Santa Barbara, USA
}

\begin{abstract}
In credit risk management, migration or transition matrices are major inputs for risk management, Credit Value-at-Risk or derivative pricing. After reviewing distance measures for migration matrices we propose some new directed difference indices to measure changes in migration behavior in a more risk-sensitive way. We quantify the changes of the classical distance measures and the new distance indices based on Moody's credit migration history from 1982-2001. We study the relationship between the difference indices, business cycle effects and changes in risk capital for exemplary credit portfolios. Our findings strongly support the usefulness of the derived distance indices and its capability to indicate cyclical behavior or changes in credit VaR. We recommend the use of the indices in risk management for internal credit and loan portfolios.
\end{abstract}

Key words: Transition Matrices, Matrix Norms, Difference Indices, Rating Migration, Credit VaR

\footnotetext{
ऋ Rachev gratefully acknowledges research support by grants from Division of Mathematical, Life and Physical Sciences, College of Letters and Science, University of California, Santa Barbara, the German Research Foundation (DFG) and the German Academic Exchange Service (DAAD). We would like to thank seminar participants at various Universities and Conferences for their valuable suggestions and helpful comments. The corresponding author appreciates the ongoing enlightening discussions with Til Schuermann and Linda Allen.

* Corresponding author. The paper provides results and extensions of the sixth and seventh chapter of my dissertation. Address: Institut für Statistik und Mathematische Wirtschaftstheorie, Universität Karlsruhe, Geb. 20.12., D-76128 Karlsruhe, Germany, tel. +49-721-608-2042, fax. +49-721-608-3811.

Email address: s.trueck@qut.edu.au (Stefan Trück).
} 


\section{Introduction}

Within the last decade, to manage and evaluate credit risk for credit portfolios especially rating based or reduced-form models have become very popular. On the one hand side this popularity is due to the straightforwardness of the approach: The models use the rating of a company as the decisive variable when it comes to evaluate the default risk of a bond or loan avoid difficulties of the structural models, Merton (1974), like determining a company's value and volatility. On the other hand, the upcoming new capital accord (Basel II) encourages banks to base their capital requirement for credit risk on internal or external rating systems (Basel Committee on Banking Supervision, 2001). This regulatory body under the Bank of International Settlements (BIS) becoming effective in 2007 aims to strengthen risk management systems of international financial institutions. As a result, the majority of international operating banks will use the so-called internal-rating based approach to determine capital requirements for their loan or bond portfolios. Default probabilities for certain rating categories but also the probabilities for moving from one rating state to another are important issues in such credit risk models. Therefore, to calculate VaR figures for internal rating systems a main input is an adequate transition matrix for the bonds or loans.

Unfortunately, due to cyclical behaviour of the economy migration matrices are not constant through time, see e.g. Nickell et al. (2000); Bangia et al. (2002); Wei (2003). Investigating the issue, Trück and Rachev (2005) show that such changes in migration or default behavior through time lead to substantial effects on risk figures for credit portfolios. Thus, to measure changes in migration behavior as well as determining adequate estimators for transi- 
tion matrices can be considered as a major issue in rating based credit risk modeling. Sometimes it may also be useful to adjust unconditional migration matrices to include additional information about the macroeconomic situation or market credit spreads, Wilson (1997b); Lando (2000); Kim (1999); Wei (2003). Since estimates or adjustments may have substantial impact on the risk figures it is important to have information on how "different" estimated, approximated or adjusted transition matrices really are from a risk perspective. This question is investigated in our paper where we develop new measures for comparison of transition matrices in rating based credit risk models. Section two gives an overview of risk management purposes where forecasting and adjusting transition matrices can be useful. Section three provides an overview on distance measures for matrices as they were suggested so far in the literature with respect to their capability of measuring differences in migration matrices from a risk perspective. Section four provides empirical results on changes in historical migration matrices using the classical distance measures and the risk-sensitive difference indices. Results for the different measures are investiagted with respect to the macroeconomic situation and credit VaR for an exemplary credit portfolio. Section five concludes and gives suggestions for future work.

\section{Transition Matrices in Credit Risk}

\subsection{Rating Based Models}

Since the introduction of the first so-called reduced form model by Fons (1994), the models can be considered as one of the major classes in credit risk model- 
Table 1

\begin{tabular}{|c|cccccccc|}
\hline & AAA & AA & A & BBB & BB & B & CCC & D \\
\hline Aaa & 92.00 & 7.00 & 1.00 & 0.00 & 0.00 & 0.00 & 0,00 & 0.00 \\
AA & 1.0 & 91.00 & 8.00 & 1.00 & 0.00 & 0.00 & 0.00 & 0.00 \\
A & 0.00 & 2.00 & 91.00 & 7.00 & 0.00 & 0.00 & 0.00 & 0.00 \\
BBB & 0.00 & 0.00 & 5.00 & 89.00 & 6.00 & 1.00 & 0.00 & 0.00 \\
BB & 0.00 & 0.00 & 0.00 & 5.00 & 85.00 & 8.00 & 1.00 & 1.00 \\
B & 0.00 & 0.00 & 0.00 & 0.00 & 6.00 & 84.00 & 4.00 & 5.00 \\
CCC & 0.00 & 0.00 & 1.00 & 2.00 & 2.00 & 14.00 & 54.00 & 27.00 \\
\hline
\end{tabular}

Standard \& Poor's 1-year transition matrix for corporate bonds, 2002.

ing. Before that most models were based on the structural approach developed by Merton (1974) calculating default probabilities and credit spreads using the value of the company as the driver of credit risk. However, in the model developed by Fons, calculating the price of credit risk the input variables were the rating of a company and historical default probabilities for rating classes. As an extension of this simple approach not only the event of default was considered but also rating changes of a company or an issued bond. Clearly, those credit events also have influence on the price of a bond. The most popular model of this category is probably the discrete-time Markovian Model by Jarrow et al. (1997) (JLT). The model incorporates possible rating upgrade, stable rating and rating downgrade (with "default" as a special event) in the reduced form approach. Downgrades or upgrades by the rating agencies are taken very seriously by market players to price bonds and loans, thus effecting the risk premium and the yield spreads. Also in one of the most popular practical implementations, CreditMetrics, historical transition matrices are used to determine Value-at-Risk figures or the price of a bond. Often, as a basis, historical transition matrices like given in table 1 are used.

In their seminal paper, JLT model default and transition probabilities by using a discrete time, time-homogenous Markov chain on a finite state space $\mathrm{S}=\{1, \ldots \ldots, \mathrm{K}\}$. The state space $\mathrm{S}$ represents the different rating classes. While 
1 is for the best credit rating, $\mathrm{K}$ represents the default case. Hence, the (KxK) one-period transition matrix is:

$$
P=\left(\begin{array}{cccc}
p_{11} & p_{12} & \cdots & p_{1 K} \\
p_{21} & p_{12} & \cdots & p_{2 K} \\
\cdots & \cdots & \cdots & \cdots \\
p_{K-1,1} & p_{K-1,2} & \cdots & p_{K-1, K} \\
0 & 0 & \cdots & 1
\end{array}\right)
$$

where $p_{i j} \geq 0$ for all $\mathrm{i}, \mathrm{j}, \mathrm{i} \neq \mathrm{j}$, and $p_{i i} \equiv 1-\sum_{\substack{j=1 \\ j \neq i}}^{K} p_{i j}$ for all i. $p_{i j}$ represents the actual probability of going to state $\mathrm{j}$ from state $\mathrm{i}$ in one time step. The default state $\mathrm{K}$ is an absorbing one. JLT also present a continuous-time approach, where the time-homogenous Markov chain is specified via a $(\mathrm{KxK})$ generator matrix:

$$
\Lambda=\left(\begin{array}{llll}
\lambda_{11} & \lambda_{12} & \cdots & \lambda_{1 K} \\
\lambda_{21} & \lambda_{22} & \cdots & \lambda_{2 K} \\
\cdots & \cdots & \cdots & \cdots \\
\lambda_{K-1,1} & \lambda_{K-1,2} & \cdots & \lambda_{K-1, K} \\
0 & 0 & \cdots & 0
\end{array}\right)
$$

where $\lambda_{i j} \geq 0$, for all $\mathrm{i}, \mathrm{j}$ and $\lambda_{i i}=-\sum_{\substack{j=1 \\ j \neq i}}^{K} \lambda_{i j}$, for $\mathrm{i}=1, \ldots . \mathrm{K}$. The off-diagonal elements represent the intensities of jumping to rating $\mathrm{j}$ from rating i. Again default is an absorbing state.

The $(\mathrm{KxK})$ t-period transition matrix under the actual probabilities is then given by

$$
P(t)=e^{t \Lambda}=\sum_{k=0}^{\infty} \frac{(t \Lambda)^{k}}{k !}=I+(t \Lambda)+\frac{(t \Lambda)^{2}}{2 !}+\frac{\left(t \Lambda^{3}\right)}{3 !}+\cdots
$$

While in most publications discrete time transition matrices are reported, 
Christensen et al. (2004); Lando and Skødeberg (2002) show that for several issues using the continous-time approach has some advantages. Using generator matrices one can obtain transition matrices for arbitrary time horizons. Due to the fact that within a time-period $t$ multiple rating changes are allowed one can get non-zero estimates for probabilities of rare events which the multinomial method estimates to be zero. Further, the continuous time framework permits generating confidence sets for default probabilities, the dependence on covariates can be tested and business cycles effects can be quantified.

In the following sections we will therefore deal both with discrete and continoustime approaches to transition matrices.

\subsection{Stability of Transition Matrices}

Due to the new Basel Capital Accord most of the European Banks will determine their regulatory capital based on an internal rating system. Therefore, they will have ratings and default probabilities for all loans and bonds in their credit portfolio. As aforementioned, historical transition matrices could be used as an input for estimating portfolio loss distributions and Value-at-Risk figures. Unfortunately, transitions of loans in internal bank portfolios behave not the same way as the transitions provided by the rating agencies Moody's or Standard \& Poor's. Further, transition matrices cannot be considered to be constant over a longer time period. Nickell et al. (2000) show that there is quite a big difference between transition matrices during a expansion of the economy and a recession. The results are confirmed by Bangia et al. (2002) who suggest that for risk management purposes it might be interesting not 
only to simulate the term structure of default but to design stress test scenarios by the observed behavior of default and transition matrices through the cycle. Trück and Rachev (2005) show that the effect of different migration behavior on exemplary credit portfolios may lead to substantial changes in expected losses, credit VaR or confidence sets for probabilities of default (PDs). During a recession period of the economy the VaR for one and the same credit portfolio was up to eight times higher than during an expansion of the economy. As a consequence, Bangia et al. (2002) suggest to extend transition matrix application to a conditional perspective using additional information on the economy or even forecast transition matrices using revealed dependencies on macro-economic indices and interest rates. To approach these issues it is a major concern to be able to judge whether one has an adequate model or forecast for a conditional or unconditional transition matrix. It rises the question what can be considered to be a "good" forecast and how forecasted rating transitions can be compared. Therefore, risk-sensitive goodness-of-fit measures are needed. We will see in later sections that the standard distance cell-by-cell metrics cannot deal with this issue.

\subsection{Finding adequate generator matrices}

If one wants to use a continuous-time approach it is important to estimate the adequate generator matrix. One way is to use the Maximum-Likelihood estimator

$$
\hat{\lambda}_{i j}=\frac{N_{i j}(T)}{\int_{0}^{T} Y_{i}(s) d s}
$$

to find the generator matrix directly. This approach only works if the exact 
time of the transition is available.

However, in internal rating systems the exact information of the time of a rating change may not be available. Often for a loan portfolio balance sheet data and rating changes are reported only once a year. Then only a discrete time (one-year) migration matrix is available. In this case, given the one-year $N \times N$ transition matrix $P$ we are interested in finding a generator matrix $\Lambda$ such that:

$$
P=e^{\Lambda}
$$

Dealing with the question if there exists such a generator matrix, the following theorem Noris (1998) can be used:

Theorem 1 If a migration matrix $P=\left(p_{i j}\right) \quad i, j=1, \cdots, K$ is strictly diagonal dominant, i.e. $p_{i i}>0.5$ for every $i$, then the log-expansion

$$
\Lambda_{n}=\sum_{k=1}^{n}(-1)^{k+1} \frac{(P-I)^{k}}{k} \quad(n \in \mathbb{N})
$$

converges to a matrix $\Lambda=\left(\lambda_{i j}\right) i, j=1, \cdots, K$ satisfying

$$
\begin{aligned}
& \text { 1. } \sum_{j=1}^{n} \lambda_{i j}=0 \text { for every } i=1, \cdots, K \\
& \text { 2. } \exp (\Lambda)=P .
\end{aligned}
$$

The convergence $\Lambda_{n} \rightarrow \Lambda$ is geometrically fast and defines a $N \times N$ matrix having row-sums of zero and satisfying $P=e^{\Lambda}$ exactly. For the proof, see Israel et al. (2000). However, often there remains the following problem: The main disadvantage of series (6) is that $\Lambda_{n}$ may converge but does not have to be a true generator matrix in economic sense, particularly it is possible that 
some off-diagonal elements are negative. From an economic viewpoint this is not acceptable because a negative entry in the generator may lead to negative transition probabilities for very short time intervals (Israel et al., 2000).

For application of the continuous-time approach, if there exists no valid generator, a procedure is necessary to provide an approximation of the continoustime transition matrix. The literature, suggests different methods to deal with this problem, see e.g. Jarrow et al. (1997) or Israel et al. (2000).

Again, the question rises what can be considered to be a "good" approximation in a sense that the value-at-risk of the portfolio is not significantly underor overestimated if the approximation is used. For this purpose adequate difference measures for migration matrices are necessary.

\subsection{Modifying Transition matrices}

Another issue when dealing with transition matrices is to adjust, re-estimate or change the transition matrix due to some economic reason. For example the matrix could be modified to obtain a risk-neutral transition matrix that matches default probabilities implied in bond prices observed in the market as it is done in the JLT model. Another issue could be to change the matrix according to some macroeconomic forecasts that will affect future transitions in the portfolio. The first model developed to explicitly link business cycles to rating transitions was in 1997 CreditPortfolio View (CPV) by Wilson (1997b,a). Kim (1999) develops a univariate model whereby ratings respond to business cycle shifts. The model is extended to a multifactor credit migration model by Wei (2003). Nickell et al. (2000) propose an ordered probit model which permits migration matrices to be conditioned on the industry, the country 
domicile, and the business cycle.

A major question is how one can judge the effect of the modifications on VaR figures and the loss distribution of the portfolio. Further, a task could be to decide based on such information which modification method is the right one to choose. Further, from our point of view so far there is no appropriate method suggested in the literature for evaluating the performance of the forecasting or adjustment procedures for migration matrices.

We conclude that there is need for measures giving information on how "different" estimated, approximated or adjusted transition matrices are from unconditional or average historical transition matrices. We will show in the next sections that classical cell-by-cell distance measures for matrices are not an optimal choice for these issues.

\section{Distance Measures for Matrices}

An important task for evaluating the goodness of our model or to compare our forecasts with actual transition matrices is to measure the distance between two matrices. In the literature one can find several measures based on cell-bycell distances, eigenvalues Geweke et al. (1986), eigenvectors Arvanitis et al. (1999), metrics based on singular-values Jafry and Schuermann (2004) or socalled risk-sensitive difference indices for matrices Trück (2004). We will give a brief summary of the measures described in the literature so far. 


\subsection{Classical Matrix Norms}

The first group to be mentioned are the classical cell-by-cell distance measures. Probably the most intuitive and prominent among this class of measures are the $L_{1}, L_{2}$ or $L_{m} a x$ metric. They can be denoted as

$$
\begin{aligned}
& D_{L_{1}}(P, Q)=\sum_{i=1}^{n} \sum_{j=1}^{n}\left|p_{i j}-q_{i j}\right|, \\
& D_{L_{2}}(P, Q)=\sqrt{\sum_{i=1}^{n} \sum_{j=1}^{n}\left(p_{i j}-q_{i j}\right)^{2}} \\
& D_{L_{\max }}(P, Q)=\max _{i, j}\left|p_{i j}-q_{i j}\right|
\end{aligned}
$$

where $n$ is the number of columns and rows as migration matrices are symmetric. The $L_{1}$ metric is used for example in Israel et al. (2000) for comparing migration matrices while Bangia et al. (2002) suggest the $L_{2}$ metric as a distance measure. The literature provides several variations and extensions of the $L_{1}$ and $L_{2}$ metric. Some of them were used solving optimization problems, e.g. in input-output analysis, Jackson and A. (2004). Most of them can be represented by a category of distance measures of the form

$$
D_{\text {weight }}(P, Q)=\sum_{i=1}^{n} \sum_{j=1}^{n} p_{i j}^{k}\left|p_{i j}-q_{i j}\right|^{p}
$$

with $k$ varying from -1 to 1 and p varying from 1 to infinity. For $k$ less than 0 , the elements $p_{i j}$ cannot be zero, or the fraction will be undefined. Note that obviously (10) does not define a metric or distance in the usual sense, see e.g. 
Rachev (1991). That is why we prefer using the term 'index', 'difference index' or 'deviation' to denote a quantity like that in (10).

Lahr (2001) suggests a so-called weighted absolute difference (WAD) measure for input-output analysis. The measure is expressed as:

$$
D_{W A D}(P, Q)=\sum_{i=1}^{n} \sum_{j=1}^{n} p_{i j} \cdot\left|p_{i j}-q_{i j}\right|
$$

Obviously $D_{W A D}(P, Q) \neq D_{W A D}(Q, P)$, so $D_{W A D}$ does not satisfy the symmetry condition. This could be guaranteed for example by defining a distance measure $D_{W A D^{\text {symm }}}=0.5 \cdot\left(D_{W A D}(P, Q)+D_{W A D}(Q, P)\right)$ or $D_{W A D^{s y m m}}=$ $\max \left(\left(D_{W A D}(P, Q), D_{W A D}(Q, P)\right)\right.$.

Matuszewski et al. (1964) suggest a different version of the absolute differences using normalized absolute differences (NAD). In this formulation, differences in large coefficients will contribute less to the value of distance than will equally sized differences in small coefficients. Clearly, this imposes a greater penalty on changes in small coefficients.

$$
D_{N A D}(P, Q)=\sum_{i=1}^{n} \sum_{j=1}^{n} \frac{\left|p_{i j}-q_{i j}\right|}{p_{i j}}
$$

The elements $p_{i j}$ cannot be zero, or the fraction will be undefined. This makes a straightforward application to transition matrices rather difficult, since it is quite likely that some migration probabilities can be zero. Also, since $D_{N A D}(P, Q) \neq D_{N A D}(Q, P)$, the symmetry condition is not satisfied as well but could be ensured by using the same procedure as it was suggested for $D_{W A D}$ 
Similar expressions for the $L^{2}$ metric are straightforward. The measures obtained are then called weighted squared differences (WSD) and normalized squared differences (NSD).

Trück (2004) shows that from a 'risk perspective' the surveyed cell-by-cell distance measures are not optimal to measure changes in transition matrices. They do not distinguish between differences in default or non-default transitions. Also there is no distinction between differences that appear in cells to the left (upgrades) or right (downgrades) of the diagonal.

\subsection{Measuring the mobility of a matrix}

Another approach is provided by Jafry and Schuermann (2004). They develop a scalar metric which captures the overall dynamic size of given matrices and contains sufficient information to facilitate meaningful comparisons between different credit migration matrices. Primarily the so-called mobility matrix $\tilde{P}$ is calculated by subtraction of the identity matrix $I$ from the original transition matrix $P$. Obviously, the identity matrix can be considered as a static migration matrix. Subtracting the identity matrix the authors conclude that only the dynamic part of the originial matrix remains.

Further, following Strang (1988) the mobility of a matrix can be captured by its so-called "amplifying power" on a state vector $x$. In Strang (1988), therefore it is suggested to use the largest singular value of a matrix as a mobility norm. However, Jafry and Schuermann (2004) conclude that the maximally-amplified vector $x$ is not representative of a feasible state vector. Thus, it is proposed to use the average of all singular values of $P$ to capture the general characteristics of $P$. The metric is defined as the average of the singular values of the mobility 
$\operatorname{matrix}^{1}$ :

$$
M_{S V D}(P)=\frac{\sum_{i=1}^{n} \sqrt{\lambda_{i}\left(\tilde{P}^{\prime} \tilde{P}\right)}}{n} .
$$

The authors show that this metric captures the so-called 'amplification factor' or the dynamic part of the migration matrix. Therefore, it approximates the average probability of migration which can be considered as a meaningful magnitude calibration for a metric. To measure the difference between two migration matrices in terms of mobility one has to calculate:

$$
D_{S V D}(P, Q)=M_{S V D}(P)-M_{S V D}(Q) .
$$

The expression (14) gives a directional deviation between two matrices in terms of the mobility or approximate averge probability of migration.

\subsection{Risk-adjusted difference indices}

A new approach to comparison of transition matrices for application to credit risk is provided in Trück (2004). The author suggests to consider changes in transition matrices from the angle of risk management and considers the following issues as helpful for developing an appropriate distance measure:

The direction of the transition (DIR): If we would like to introduce a risksensitive measure for differences between migration matrices, the direction of the shift in probability mass matters. If more mass is shifted to upgrades there

$\overline{1}$ The singular values of $\tilde{P}$ are equal to the eigenvalues $\lambda_{i}$ of $\tilde{P}^{\prime} \tilde{P}$. 
will be less defaults to expect and a shift of the probability mass to downgrades will end in a higher risk for the credit portfolio.

Capturing transitions to the default state (TD): Since defaults can be considered to be the major risk for the companies in the portfolio, a risk-sensitive distance measure has to seperate the default columns from the others. Changes in this column have to receive a clearly higher weight. We suggest different multipliers depending on the dimension $n$ of the transition matrix P. Possible multipliers could be $n, 2 n, n^{2}$ or $\exp (n)$.

Capturing the probability mass in a cell $(\boldsymbol{P} \boldsymbol{M})$ : To capture the probability of transition in an individual cell, weighted difference indices NAD, NSD, WAD and WSD can be used. Thus, the probability mass in the cell of the original matrix is considered as a weight either by multiplying or dividing by $p_{i j}$.

Capturing the migration deviation ( $M D$ ): To capture the difference between close and far migrations a coefficient $(i-j)$ for measuring the distance between the two rating states is used.

Based on these criteria, following Trück (2004), we suggest to use weights for differences in a cell $(i, j)$ between transition matrices $P$ and $Q$ according to:

$$
\begin{aligned}
& d_{1}(i, j)=(i-j) \cdot\left(p_{i j}-q_{i j}\right) \\
& d_{2}(i, j)=\frac{(i-j)}{p_{i j}} \cdot\left(p_{i j}-q_{i j}\right)
\end{aligned}
$$

Obviously these weights include the mentioned criteria DIR and MD. Far transitions get a higher weight than near transitions and the sign multiplied 
by the difference between the cells leads to higher numbers of the measure if more probability mass is shifted to the right hand side of the diagonal. $d_{2}$ is simply $d_{1}$ including normalized weights (only for the cells where $p_{i j} \neq 0$ ) and therefore, taking into account the original transition probability PM where the deviation occurred.

$$
\begin{aligned}
& d_{3}(i, j)=(i-j) \cdot \operatorname{sign}\left(p_{i j}-q_{i j}\right) \cdot\left(p_{i j}-q_{i j}\right)^{2} \\
& d_{4}(i, j)=\frac{(i-j)}{p_{i j}} \operatorname{sign}\left(p_{i j}-q_{i j}\right) \cdot\left(p_{i j}-q_{i j}\right)^{2}
\end{aligned}
$$

$d_{3}$ and $d_{4}$ use the squared differences instead of the actual difference. Therefore, the sign of the deviation has to be added to the formula as well.

We are well aware of the fact that measuring the cell-by-cell differences of two migration matrices with $d_{1}, d_{2}, d_{3}, d_{4}$ will give us difference indices that cannot satisfy the required conditions for a metric. This seems to be critical from a mathematical point of view at first sight. However, we will show in later sections that the advantages over standard deviation measures for matrices or norms are so clear cut that we are willing to accept this. From the perspective of application for risk management purposes we will show the superior properties in the next section.

Based on the weights for individual cells of two migration matrices we then define the following indices measuring the difference between migration matrices in terms of risk:

$$
D_{k}(P, Q)=\sum_{i=1}^{n} \sum_{j=1}^{n} d_{k}(i, j), \quad k=1,2,3,4 .
$$

The credit event with the most influence on the loss in the portfolio is a 
default. Therefore, changes in the default of a migration matrix should obtain a different weight than deviances in the other cells. To capture also the criteria TD we use the following indices:

$$
D_{5}(P, Q)=\sum_{i=1}^{n} \sum_{j=1}^{n-1} d_{3}(i, j)+\sum_{i=1}^{n} n \cdot d_{3}(i, n)
$$

and

$$
D_{6}(P, Q)=\sum_{i=1}^{n} \sum_{j=1}^{n-1} d_{3}(i, j)+\sum_{i=1}^{n} n^{2} \cdot d_{3}(i, n)
$$

$D_{5}$ and $D_{6}$ use squared differences between the cells and apply to the default column a multiplier of $n$ or $n^{2}$. It is straightforward to also use absolute differences between the cells and define:

$$
D_{7}(P, Q)=\sum_{i=1}^{n} \sum_{j=1}^{n-1} d_{1}(i, j)+\sum_{i=1}^{n} n \cdot d_{1}(i, n)
$$

and

$$
D_{8}(P, Q)=\sum_{i=1}^{n} \sum_{j=1}^{n-1} d_{1}(i, j)+\sum_{i=1}^{n} n^{2} \cdot d_{1}(i, n) .
$$

Again for the default columns the weights $n$ and $n^{2}$ are applied.

In Trück (2004) it is shown that some of the difference indices $D_{1}(P, Q)-$ $D_{8}(P, Q)$ are highly correlated to changes in Value-at-Risk figures due to shifts in probability mass of transition matrices. However, the investigation is based on simplified portfolios and migration matrices only. In the next section we will now calculate the introduced difference indices for a 20-year history of Moody's migration matrices. 


\section{Empirical Results}

In this section we will examine the changes in the distance indices on empirical data. For the analysis we use Moody's credit migration history from 1982-2001. We will also study the relationship between the difference indices and changes in risk capital for an exemplary credit portfolio. Our findings strongly support the usefulness of the derived distance indices.

\subsection{Changes in the Distance Measures Through Time}

First we take a glance at the changes or deviations of transition matrices to a reference migration matrix through time. Special focus is set on the behavior of the directed distance measures during periods of economic expansion and recession. It should be assumed that a distance measure that is able to capture also the direction of the deviation will give positive values for recession periods like in 1990-1991 or in 2001 when there are more downgrades or migrations to the right hand side of the diagonal. On the other hand a risk sensitive distance index should rather give negative values for periods of economic expansions with only few defaults and migrations rather to the left hand side of the diagonal.

A first step was to calculate an average one-year transition matrix $\bar{P}$ for the considered period. Based on a 20-year observation period we calculated the average one-year transition matrix. We further examined the difference between the average one-year transition matrix $\bar{P}$ and the observed one-year transition matrices $P_{1982}-P_{2001}$. Thus, according to the introduced indices $D$ in the previous section, we calculated the differences between two migration 


\begin{tabular}{|c|ccccccc|c|}
\hline & Aaa & Aa & A & Baa & Ba & B & C & D \\
\hline Aaa & 0.9276 & 0.0661 & 0.0050 & 0.0009 & 0.0003 & 0.0000 & 0.0000 & 0.0000 \\
Aa & 0.0064 & 0.9152 & 0.0700 & 0.0062 & 0.0008 & 0.0011 & 0.0002 & 0.0001 \\
A & 0.0007 & 0.0221 & 0.9137 & 0.0546 & 0.0058 & 0.0024 & 0.0003 & 0.0005 \\
Baa & 0.0005 & 0.0029 & 0.0550 & 0.8753 & 0.0506 & 0.0108 & 0.0021 & 0.0029 \\
Ba & 0.0002 & 0.0011 & 0.0052 & 0.0712 & 0.8229 & 0.0741 & 0.0111 & 0.0141 \\
B & 0.0000 & 0.0010 & 0.0035 & 0.0047 & 0.0588 & 0.8323 & 0.0385 & 0.0612 \\
C & 0.0012 & 0.0000 & 0.0029 & 0.0053 & 0.0157 & 0.1121 & 0.6238 & 0.2389 \\
D & 0.0000 & 0.0000 & 0.0000 & 0.0000 & 0.0000 & 0.0000 & 0.0000 & 1.0000 \\
\hline
\end{tabular}

Table 2

Average one-year transition matrix of Moody's corporate bond ratings for the period 1982-2001.

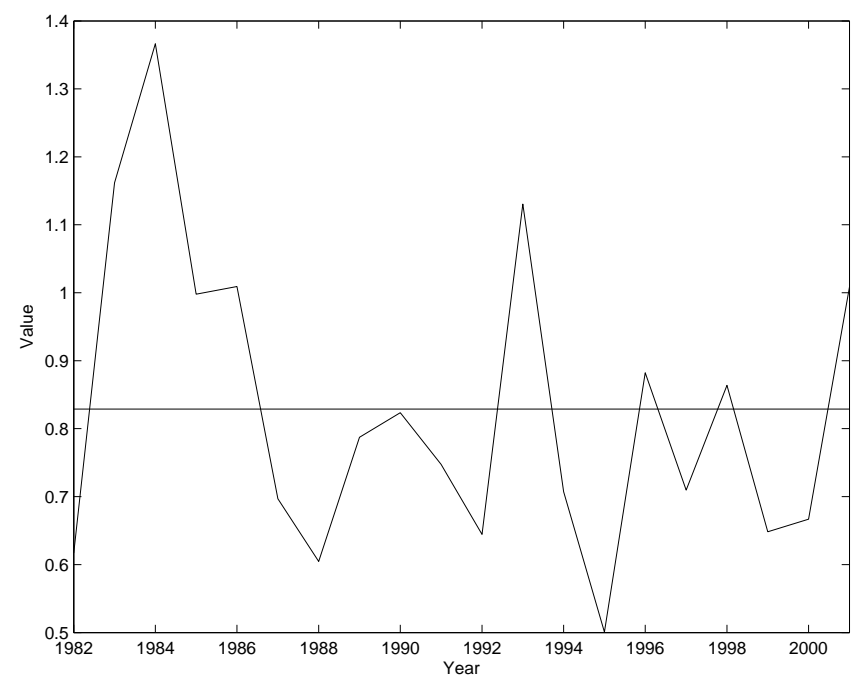

Fig. 1. Distances between the average Moody's corporate bond ratings transition matrix 1982-2001 and the one-year migration matrices for the $L_{1}$ norm.

matrices according to:

$$
\delta_{P_{t}, \bar{P}}=D\left(P_{t}, \bar{P}\right) \quad \text { for } \quad t=1982, . ., 2001
$$

Additionally we also calculated the differences according to the (weighted) cell-by-cell distance measures and standard matrix norms, the SVD 'metric' measuring the mobility of migration matrices and the ad-hoc risk sensitive difference measures D1-D8. The results are displayed in figures $1-5$.

Obviously, the distance measures based on the $L_{1}, L_{2}$ and $L_{\max }$ norm are not able to provide an adequate index for comparing transition matrices from a risk 


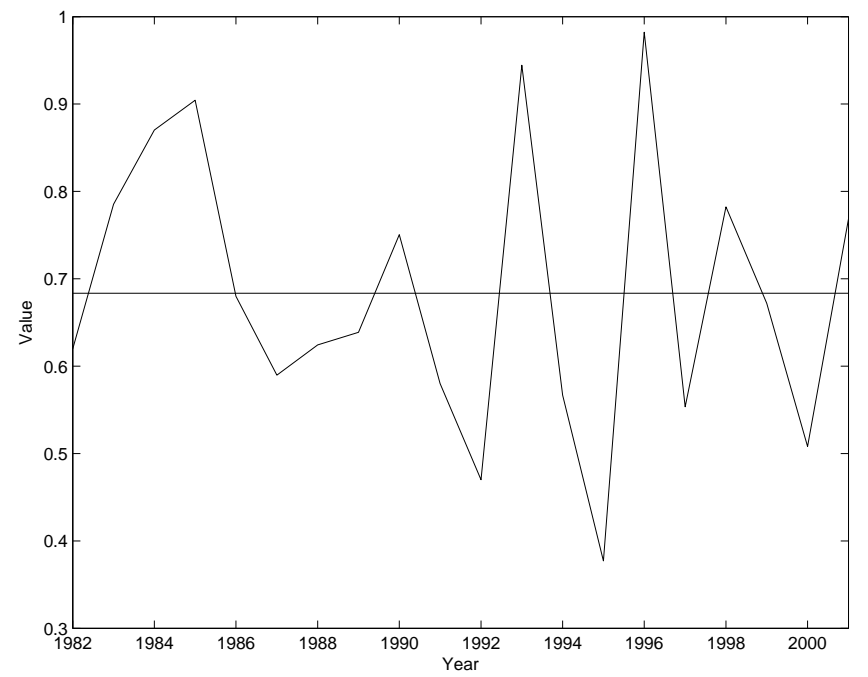

Fig. 2. Distances between the average Moody's corporate bond ratings transition matrix 1982-2001 and the one-year migration matrices for the $N S D_{\text {symm }}$ index.

perspective. While the largest deviances from the average transition matrix according to the $L_{1}$ and $L_{2}$ metric happen in the years 1983, 1984 and 1993, these years cannot be considered to have extraordinary high default rates or a particularly high number of rating up- or downgrades that would lead to a large difference for a portfolio also from a risk perspective. Vice versa, the periods of recession 1990-1991 or 2001 are not classified to be very different from the average transition matrix by the cell-by-cell distance indices usually suggested in the literature.

Figure 2 displays the results for a representative of the weighted cell-by-cell distance indices. Here the $N S D_{\text {symm }}$ distance index was used, however, the results are not very much different for the other indices $W A D^{\text {symm }}, N A D^{\text {symm }}$ and $W S D^{\text {symm }}$. Graphs for two of the other weighted cell-by-cell distance measures can be found in the appendix. We find that the largest deviations from the average transition matrix can be observed for the years 1985, 1993 and 1996. Considering Moody's migration data these are years with rather lower default rates or downgrades. Vice versa, the years of economic contraction or 


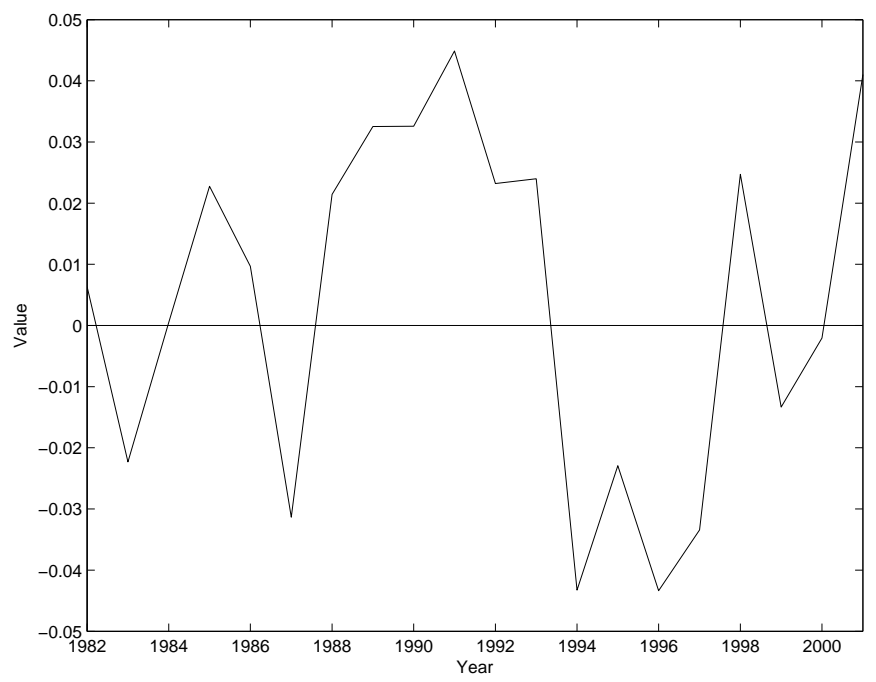

Fig. 3. Distances between the average Moody's corporate bond ratings transition matrix 1982-2001 and the one-year migration matrices for the $S V D$ metric.

distress for the financial industry 1990, 1991 or 2001 cannot be identified as showing large deviations from the average transition matrix.

The next candidate to detect differences between migration matrices is the SVD metric suggested by Jafry and Schuermann (2004). The appropriateness of the SVD metric is pointed out by the conducted empirical study. Periods of financial distress like in 2001 or 1991 in general show large deviations from the average transition matrix. Since we defined the deviation between two matrices using the SVD metric according to

$$
D_{S V D}(P, Q)=M_{S V D}(P)-M_{S V D}(Q)
$$

positive deviations like for the year 2001 indicate that there is more mobility in the matrix. This is consistent with the idea that in times of macroeconomic instability more probability mass is spread to the left and right of the diagonal and many downgrades can be observed. On the other hand during periods of only few defaults and downgrades like in 1996 or 1983 where the risk capital of the portfolio was considered to be very low also clearly lower values for the 


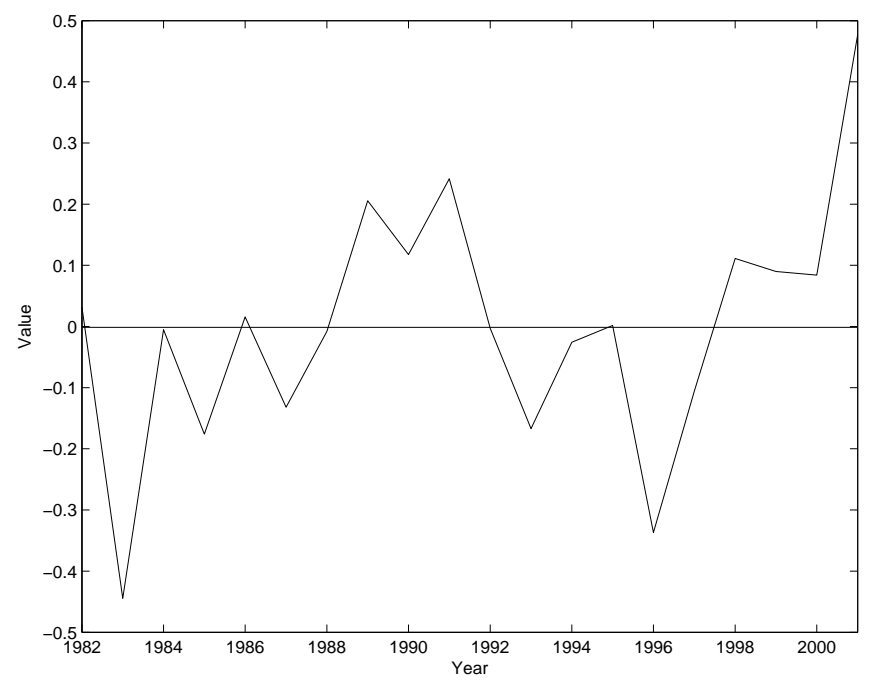

Fig. 4. Distances between the average Moody's corporate bond ratings transition matrix 1982-2001 and the one-year migration matrices for the $D_{5}$ metric.

SVD metric could be observed.

Finally, we consider changes in migration matrices using the derived risksensitive distance indices $D_{1}, \ldots, D_{8}$. The good results for the simplified migration matrices and portfolio in Trück (2004) were promising but not conducted on real empirical data. Taking a first glance at the graphs for two selected distance indices $D_{5}$ and $D_{8}$ we find that there is a strong connection between the state of the economy and the differences between the considered migration matrices according to the index. The figures for the other risk sensitive indices look very similar - some of the are displayed in the appendix, see figures 7 and 8. Another positive outcome is that the average distance between the indices and the average migration matrix is close to zero in most cases. This could not be expected naturally since the weighting procedure is not conducted in a way that could guarantees such averaging. 


\begin{tabular}{|c|ccccccc|}
\hline Rating & Aaa & Aa & A & Baa & Ba & B & Caa \\
\hline No. & 11 & 106 & 260 & 299 & 241 & 95 & 148 \\
Average Exposure (Mio. Euro) & 20 & 15 & 15 & 10 & 10 & 5 & 5 \\
\hline
\end{tabular}

Table 3

Ratings and exposures for the considered Credit Portfolio

\subsection{Credit VaR and Matrix Difference Indices}

We will now consider the link between estimated credit VaR figures of an exemplary loan portfolio and changes in migration matrices according to the distance measures of the previous sections. Following Trück and Rachev (2005), we consider an internal loan portfolio of an international operating major bank consisting of 1120 companies. The average exposure is dependent on its rating class. In the considered portfolio higher exposures could be observed in higher rating classes while companies with a non-investment grade rating Baa, B or Caa the average exposures were between 5 Mio. and 10 Mio. Euro. The distribution of ratings and average exposures in the considered rating classes of the loan portfolio is displayed in table 3 .

Further, for each of the simulated years we use the same rating distribution for the portfolio to keep the figures comparable. We also used an average yearly recovery rate of $R=0.45$ for all companies. This is clearly a simplification of real recovery rates, however due to not having any information on the seniority of the considered loans this is an adequate assumptions for empirical recovery rates. Simulated VaR figures showed great variation. The results can be found in the appendix.

Correlations for $L_{1}, L_{2}$ and $L_{\max }$ vary between -0.25 and -0.28 indicating a rather weak tendency to link changes in migration matrices to credit risk. This confirms our more theoretical results from the previous section. We conclude that these criteria for measuring the similarity of migration matrices may be 


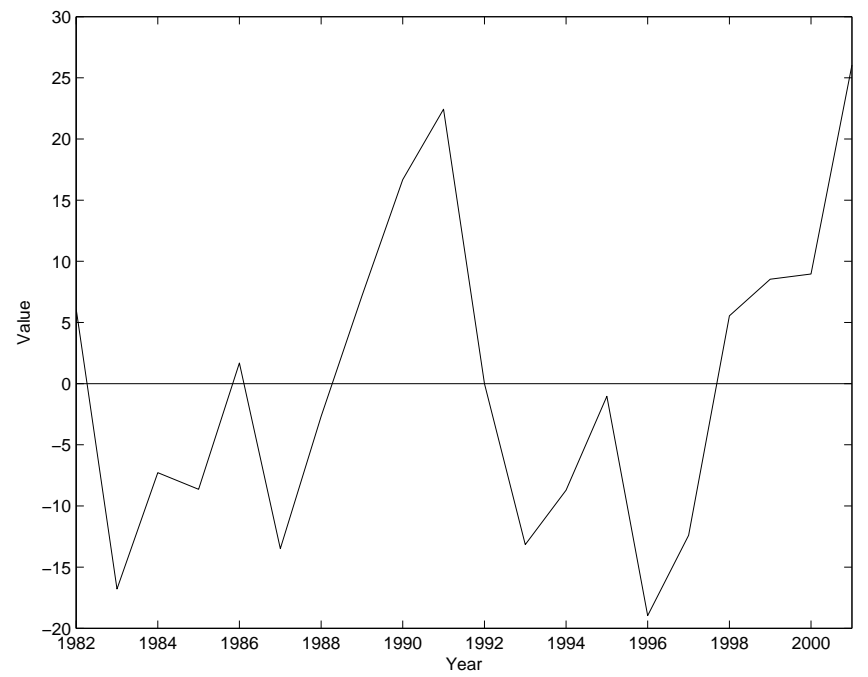

Fig. 5. Distances between the average Moody's corporate bond ratings transition matrix 1982-2001 and the one-year migration matrices for the $D_{8}$ metric.

adequate from a mathematical point of view but is not suitable for risk management purposes. It should be pointed out that this finding affects the results on goodness-of-fit for migration matrix comparison of several publications and empirical studies including some of the most prominent papers in this area by Israel et al. (2000); Bangia et al. (2002).

Results are not much better for the correlation coefficients between VaR figures and the weighted cell-by-cell distance indices. Correlations are the highest for the weighted-absolute differences $W A D^{\text {symm }}$ with 0.29 on average and the lowest for the index based on the normalized absolute differences $N A D^{\text {symm }}$ with 0.09 on average. There is no significant improvement compared to the classical cell-by-cell distance measures. This is also an important result since in the empirical study of Wei (2003) on forecasting performance of models using conditional migration matrices the absolute percentage deviation and therefore, a performance criteria based on weighted cell-by-cell distance metrics is used. We conclude that also these results should be reconsidered using different performance criteria. 
Table 4

\begin{tabular}{|c|ccccccc|}
\hline$\rho$ & $L_{1}$ & $L_{2}$ & $L_{\max }$ & $W A D$ & $N A D$ & $W S D$ & $N S D$ \\
\hline Var $_{0.95}$ & -0.257 & -0.251 & -0.267 & -0.288 & 0.089 & -0.2392 & -0.2730 \\
Var $_{0.99}$ & -0.259 & -0.258 & -0.275 & -0.290 & 0.092 & -0.244 & -0.272 \\
$E S_{0.95}$ & -0.258 & -0.254 & -0.271 & -0.289 & 0.092 & -0.240 & -0.274 \\
$E S_{0.99}$ & -0.258 & -0.258 & -0.278 & -0.289 & 0.102 & -0.240 & -0.271 \\
\hline
\end{tabular}

Correlation between (weighted) cell-by-cell distance measures and Value-at-Risk and Expected Shortfall for the considered loan portfolio through the business cycle.

For the SVD metric, correlations with determined VaR figures vary between 0.68 and 0.70 what can be considered as superior to the results of the considered cell-by-cell distance measures. The SVD metric provides a distance index for migration matrices that can also be used for determination of the risk inherent in a migration matrix. However, this is only true as long as the thesis holds that in times of business cycle troughs more mass is spread away from the diagonal while for times of expansion more mass can be found within the diagonal of migration matrices. It should also be noted that the SVD metric is not able to distinguish between transitions to the left or right hand side of the diagonal.

Finally we consider the link between estimated credit VaR figures and changes in migration matrices using the derived risk-sensitive distance indices $D_{1}, \ldots, D_{8}$. We observe that for the indices $D_{1}, \ldots, D_{4}$ that do not include the special weight on the default column the results are good but not outstanding. Correlation coefficients vary between 0.66 and 0.84 giving the worst result for the index $D_{2}$ using

$$
d_{2}(i, j)=\frac{(i-j)}{p_{i j}} \cdot\left(p_{i j}-q_{i j}\right)
$$

It seems as if the normalized absolute differences - and therefore criteria PM - do not provide adequate weights to determine the changes of the considered migration matrices. Also for the weighted cell-by-cell indices the $N A D^{\text {symm }}$ 
criteria showed the lowest correlation. Without inclusion of criteria TD the best results with a correlation greater then 0.8 is obtained $D_{1}$ using simply the measure of migration distance MD. Finally, the best results for linking changes in migration matrices to determined VaR figures provide the indices $D_{5}, \ldots, D_{8}$. Correlations are higher than 0.9 indicating a very strong linear relationship between the used distance indices and risk capital. For the difference indices $D_{7}$ and $D_{8}$, observed correlations are even close to 1 . It seems as if for the considered data using a squared weight of dimension $n^{2}$ for the default column like in $D_{8}$ captures almost perfectly the differences in migration matrices as they affect changes in the risk for a credit portfolio. Thus, we define the difference between a matrix $P$ and $Q$ according to the weighted index to default (WID) as:

$$
W I D(P, Q)=\sum_{i=1}^{n} \sum_{j=1}^{n-1}(i-j) \cdot\left(p_{i j}-q_{i j}\right)+n^{2} \sum_{i=1}^{n}(i-n) \cdot\left(p_{i n}-q_{i n}\right) .
$$

Based on these results we could even estimate a regression model giving information on how changes in the difference index affect the VaR. We did this exemplary for the $V a R_{95 \%}$ of the considered portfolio and the difference index $D_{8}$. The result is an equation of the following form:

$$
\operatorname{VaR}_{95 \%, t}=138.7675+4.7110 \cdot W I D\left(P_{t}, \bar{P}\right)+\epsilon_{t}
$$

giving a goodness-of-fit statistic $R^{2}$ of 0.9754 .

This can be considered as a clear improvement for evaluating the adequacy of adjustment methods for migration matrices. Our findings support the usefulness of the derived ad-hoc distance indices and its capability to indicate changes in credit VaR. 


\begin{tabular}{|c|ccccccccc|}
\hline$\rho$ & $S V D$ & $D_{1}$ & $D_{2}$ & $D_{3}$ & $D_{4}$ & $D_{5}$ & $D_{6}$ & $D_{7}$ & $D_{8}$ \\
\hline Var $_{0.95}$ & 0.691 & 0.834 & 0.677 & 0.759 & 0.711 & 0.924 & 0.916 & 0.981 & 0.988 \\
Var $_{0.99}$ & 0.690 & 0.842 & 0.683 & 0.765 & 0.711 & 0.921 & 0.911 & 0.982 & 0.987 \\
$E S_{0.95}$ & 0.690 & 0.838 & 0.680 & 0.762 & 0.714 & 0.923 & 0.914 & 0.981 & 0.987 \\
$E S_{0.99}$ & 0.689 & 0.843 & 0.684 & 0.767 & 0.715 & 0.921 & 0.910 & 0.980 & 0.984 \\
\hline
\end{tabular}

Table 5

Correlation between SVD metric, derived distance indices and Value-at-Risk and Expected Shortfall for the considered loan portfolio through the business cycle.

\section{Conclusions and Future Work}

This paper suggests the use of a new class of directed difference indices for measuring changes in credit migration matrices. Transition matrices are major inputs for risk management, credit Value-at-Risk or derivative pricing in rating based credit risk models. After illustrating the necessity of adequate measures for comparing transition matrices we provided an overview on distance indices for matrices as they were suggested in the literature so far. A special focus was set on their capability to measure differences in migration matrices from a risk perspective. In an empirical study we examined the changes of the distance indices based on Moody's credit migration history from 1982-2001. Results for the different measures were investigated with respect to the macroeconomic situation and credit VaR for an exemplary credit portfolio. Our findings support the usefulness of the derived ad-hoc distance indices and its capability to indicate business cycle effects or changes in credit VaR.

We conclude that the new class of ad-hoc difference indices for migration matrices provides astonishing good results in an empirical study. Considering a 20-year history of Moody's migration matrices we find that there is a significant linear relationship between determined risk figures for exemplary credit portfolios and the derived indices. It should be pointed out that particularly in contrast to the norms or indices suggested in the literature so far for comparison of migration matrices, the directed difference indices perform sig- 
nificantly better. As a consequence, we recommend further tests of the indices on empirical data of banks' internal rating systems.

As it was pointed out in previous sections, so far there is no commonly accepted method for adjustments or forecasts of migration matrices based on credit spreads or macroeconomic variables. In addition, the derived ad-hoc difference indices could be used for evaluating the performance of different forecasting or adjustment procedures. We found that using difference measures like the SVD mobility metric or the ad-hoc risk sensitive difference indices seemed appropriate for risk management purposes. We point out that using the class of risk-sensitive indices could also provide substantial information on measuring the forecast error in terms of risk capital. This issue should be investigated more thoroughly in future work.

\section{Appendix}

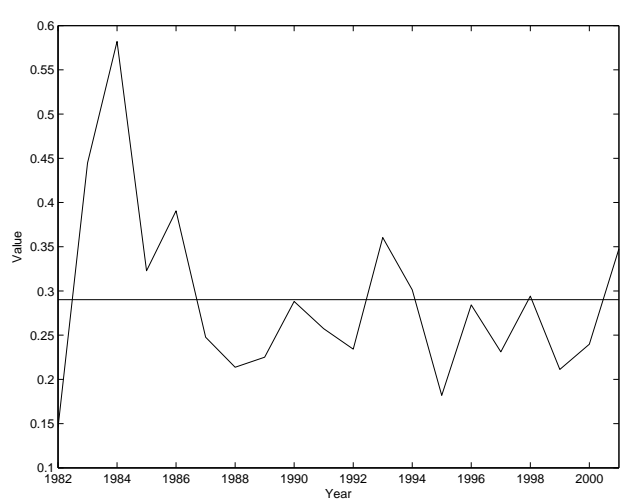

(a) WAD

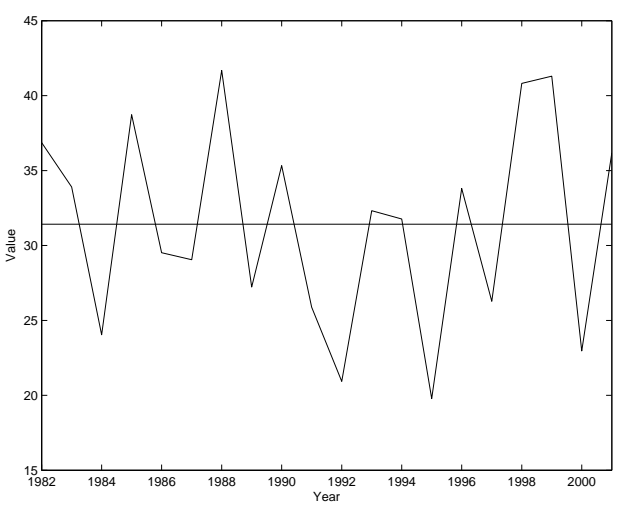

(b) NAD

Fig. 6. Distances between the average Moody's corporate bond ratings transition matrix 1982-2001 and the one-year migration matrices ( $W A D_{\text {symm }}$ and $N A D_{\text {symm }}$ ). 


\begin{tabular}{|c|c|c|c|c|c|c|c|c|c|c|}
\hline Year & 1982 & 1983 & 1984 & 1985 & 1986 & 1987 & 1988 & 1989 & 1990 & 1991 \\
\hline VaR ${ }_{0.95,1 \text { year }}$ & 191.25 & 49.50 & 108.00 & 92.25 & 132.75 & 72.00 & 135.00 & 185.62 & 217.12 & 227.25 \\
\hline $\operatorname{Va} R_{0.99,1 \text { year }}$ & 209.25 & 58.50 & 120.37 & 105.75 & 148.50 & 79.87 & 148.50 & 200.25 & 231.75 & 243.00 \\
\hline Year & 1992 & 1993 & 1994 & 1995 & 1996 & 1997 & 1998 & 1999 & 2000 & 2001 \\
\hline $\operatorname{VaR}_{0.95,1 \text { year }}$ & 135.00 & 81.00 & 101.25 & 139.50 & 45.00 & 76.50 & 175.50 & 174.37 & 177.75 & 258.75 \\
\hline $\operatorname{VaR} R_{0.99,1 \text { year }}$ & 209.25 & 58.50 & 120.37 & 105.75 & 148.50 & 79.87 & 148.50 & 200.25 & 231.75 & 243.00 \\
\hline
\end{tabular}

Table 6. 1-year 95\%- and 99\%-VaR for the exemplary portfolio loan portfolio. The figures are based on a continuous-time simulation approach Trück and Rachev (2005) based on Moody's historical migration matrices from 1982-2001. 


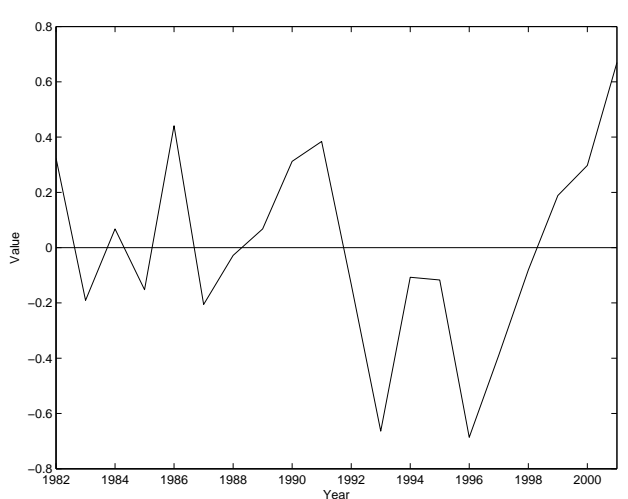

(a) D1

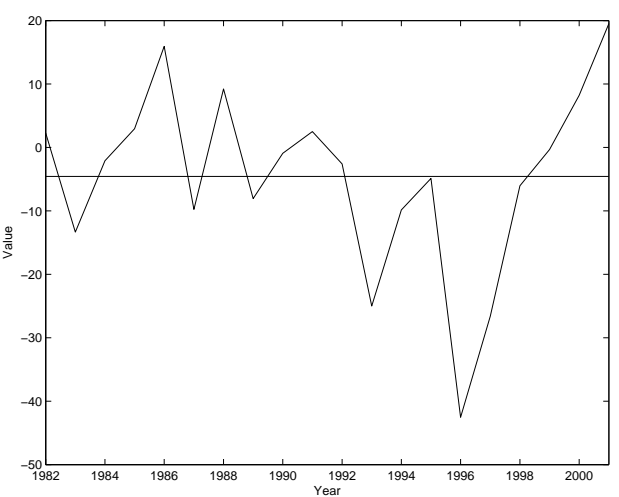

(b) D2

Fig. 7. Distances between the average Moody's corporate bond ratings transition matrix 1982-2001 and the one-year migration matrices $\left(D_{1}\right.$ and $\left.D_{2}\right)$.

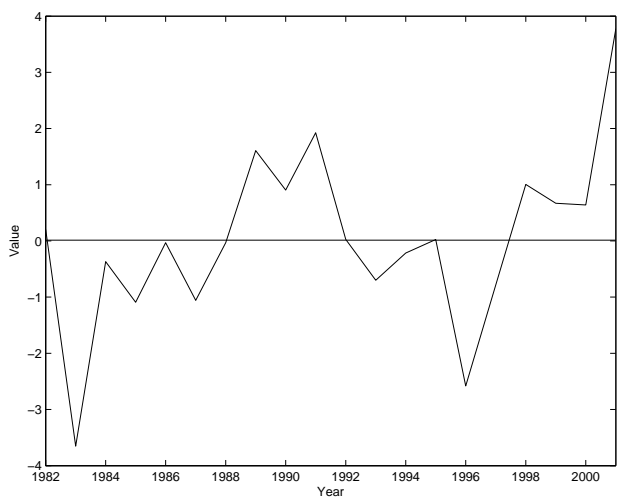

(a) D6

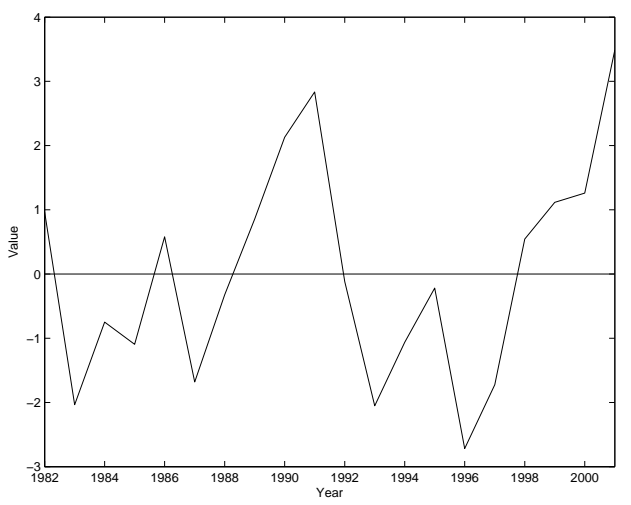

(b) D7

Fig. 8. Distances between the average Moody's corporate bond ratings transition matrix 1982-2001 and the one-year migration matrices $\left(D_{6}\right.$ and $\left.D_{7}\right)$.

\section{References}

Arvanitis, A., Gregory, J., Laurent, J.-P., 1999. Building Models for Credit Spreads. Journal of Derivatives 6, 27-43.

Bangia, A., Diebold, F., Kronimus, A., Schagen, C., Schuermann, T., 2002. Ratings Migration and the Business Cycle, with Application to Credit Portfolio Stress Testing. Journal of Banking and Finance 26, 445-474.

Basel Committee on Banking Supervision, 2001. The new Basel Capital Accord, Second Consultative Document.

Christensen, J., Hansen, E., Lando, D., 2004. Confidence Sets for Continuous 
Time Rating Transition Probabilities. Journal of Banking and Finance 28. Fons, J. S., 1994. Using Default Rates to Model the Term Structure of Credit Risk. Financial Analysts Journal Sept-Oct, 25-33.

Geweke, J., Marshall, R., G., Z., 1986. Mobility Indices in Continuous Time Markov Chains. Econometrica 54, 1407-1423.

Israel, R., Rosenthal, J., Wei, J., 2000. Finding Generators for Markov Chains via Empirical Transition Matrices, with Application to Credit Ratings. Mathematical Finance 11, 245-265.

Jackson, R., A., M., 2004. Alternative Input-Output Matrix Updating Formulations. Economic Systems Research 16.

Jafry, Y., Schuermann, T., 2004. Measurement, Estimation and Comparison of Credit Migration Matrices. Journal of Banking and Finance 28, 2603-2639. Jarrow, R., Lando, D., Turnbull, S., 1997. A Markov Model for the Term Structure of Credit Risk Spreads. Review of Financial Studies 10, 481-523. Kim, J., 1999. Conditioning the Transition Matrix. Risk Credit Risk Special Report, 37-40.

Lahr, M., 2001. A Strategy for Producing Hybrid Regional Input-Output Tables. In: Lahr, M., Dietzenbacher, E. (Eds.), Input-Output Analysis: Frontiers and Extensions. Palgrave, pp. 182-191.

Lando, D., 2000. Some Elements of Rating-Based Credit Risk Modeling. Wiley, pp. 193-215.

Lando, D., Skødeberg, T., 2002. Analyzing Rating Transitions and Rating Drift with Continuous Observations. Journal of Banking and Finance 26.

Matuszewski, T., P., P., Sawyer, J. A., 1964. Linear Programming Estimates of Changes in Input-Output Coefficients. Canadian Journal of Economics and Political Science 30, 203-210.

Merton, R., 1974. On the Pricing of Corporate Debt: The Risk Structure of 
Interest Rates. Journal of Finance 29, 449-470.

Nickell, P., W., P., Varotto, S., 2000. Stability of Rating Transitions. Journal of Banking and Finance 1-2, 203-227.

Noris, J., 1998. Markov Chains. Cambridge University Press.

Rachev, S., 1991. Probability Metrics and the Stability of Stochastic Models. Wiley, Chichester, New York.

Strang, G., 1988. Linear Algebra and its Applications. Thomson.

Trück, S., 2004. Measures for Comparing Transition Matrices from a Value-atRisk Perspective. Working Paper, Institute of Statistics and Mathematical Economics, University of Karlsruhe.

Trück, S., Rachev, T., 2005. Credit Portfolio Risk and PD Confidence Sets through the Business Cycle. Journal of Credit Risk 1 (4).

Wei, J., 2003. A Multi-Factor, Credit Migration Model for Sovereign and Corporate Debts. Journal of International Money and Finance 22, 709-735.

Wilson, T., 1997a. Measuring and Managing Credit Portfolio Risk. Mckinsey \& company.

Wilson, T., 1997b. Portfolio Credit Risk I/II. Risk 10. 